\title{
The first case of genetically confirmed sparganosis (Spirometra erinaceieuropaei) in European reptiles
}

\author{
Eliza Kondzior ${ }^{1,2} \cdot$ Małgorzata Tokarska $^{1} \cdot$ Rafał Kowalczyk $^{1} \cdot$ Iwona Ruczyńska $^{1}$ - Wojciech Sobociński ${ }^{2}$. \\ Marta Kołodziej-Sobocińska ${ }^{1}$
}

Received: 12 July 2018 / Accepted: 4 September 2018 / Published online: 15 September 2018

(C) The Author(s) 2018

\begin{abstract}
Sparganosis is a zoonosis caused by the spargana (larvae) of Spirometra sp. (Diphyllobothriidae). Reptiles are particularly important vectors for the transmission of this parasite in Asia; however, their role in sparganosis spread in European wildlife is unrecognized. We investigated the infection of reptiles with Spirometra sp. in NE Poland, where several mammalian hosts have been identified recently and in the past. Of the 59 dead reptiles, plerocercoids were found in two grass snakes (Natrix natrix) from the Białowieża Primeval Forest (BPF). The Spirometra erinaceieuropaei species was genetically confirmed using the evolutionary conserved nuclear $18 \mathrm{~S}$ rRNA gene, and then compared to GenBank deposits. The sequences were identical to previously investigated Spirometra sp. found in Eurasian badger and wild boar from BPF. Our finding is the first genetically confirmed record of Spirometra sp. in reptiles in Europe. Since reptiles are often a component of mammalian diet, they can be a source of Spirometra tapeworm infection in European wildlife; however, further studies are needed to investigate the prevalence of infection in reptiles and other non-mammalian hosts.
\end{abstract}

Keywords Natrix natrix $\cdot$ Sparganum $\cdot$ Plerocercoid $\cdot$ Non-mammalian host $\cdot$ Zoonosis $\cdot$ DNA analyses

\section{Introduction}

Sparganosis is a food- and water-borne disease still littleknown in Europe. Most research regarding it has been conducted in Asia, where sparganosis is a serious danger for public health (Wang et al. 2011, 2014; Hong et al. 2016). It is caused by spargana (plerocercoids) - the second larval stadium of the tapeworm Spirometra sp. (Diphyllobothriidae); however, knowledge of the Spirometra sp. life cycle is still limited. Adult Spirometra sp. reproduces mainly in the intestines of felids and canids such as the Eurasian lynx (Lynx lynx)

Handling Editor: Julia Walochnik

Marta Kołodziej-Sobocińska

mksobocinska@ibs.bialowieza.pl

1 Mammal Research Institute, Polish Academy of Sciences, 17-230 Białowieża, Poland

2 Institute of Biology, University of Białystok, 15-245 Białystok, Poland and wolf (Canis lupus) (Furmaga 1953; KołodziejSobocińska et al. 2018). Eggs are shed with animal feces. The parasite's first intermediate hosts are copepods (Cyclops sp.) - planktonic crustaceans in which procercoids (the first larval stadium) develop. The second intermediate or paratenic hosts can be vertebrates, such as amphibians, reptiles, birds, or mammals (including human) (Wongkulab et al. 2011; Hong et al. 2016). Procercoids develop into plerocercoids which settle in organs and tissues of intermediate hosts (Bearup 1953). The life cycle of Spirometra sp. may also include paratenic hosts, in which spargana once more settle in the tissues after passing through the intestinal wall (Wongkulab et al. 2011). These hosts, however, are not necessary for the cycle's completion.

Two species of Spirometra were found in Europe: $S$. erinaceieuropaei, found in several studies in Europe and Asia (Wang et al. 2011; Kołodziej-Sobocińska and Miniuk 2018), and S. janickii, described for the first time in the Białowieża Primeval Forest (BPF) (north-eastern Poland) in mammalian hosts such as the Eurasian lynx, wolf, red fox (Vulpes vulpes), and common shrew (Sorex 


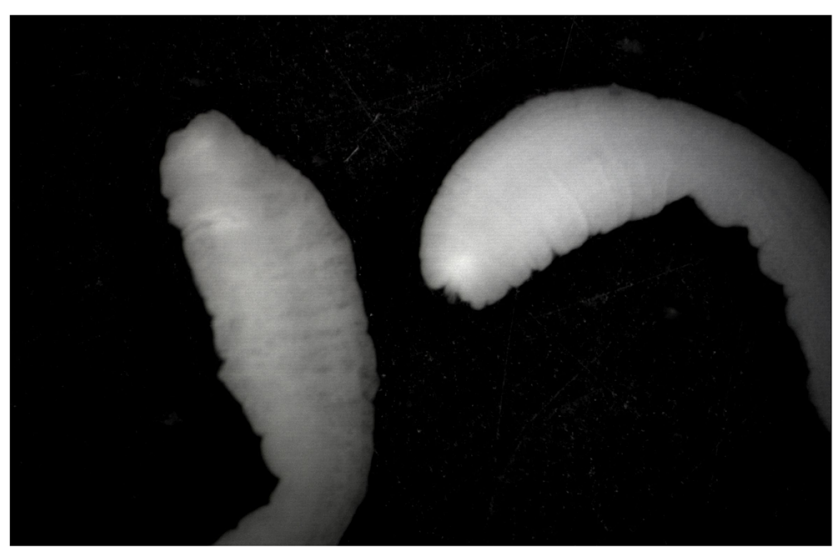

Fig. 1 Plerocercoid larvae of Spirometra sp. isolated from grass snake (Natrix natrix) subcutaneous tissue (photo: M. Kołodziej-Sobocińska)

araneus) in the 1950s (Furmaga 1953). The second species has never been confirmed in other studies; thus, its taxonomic status remains problematic. In recent years, tapeworms have been found in other hosts in BPF, including the wild boar (Sus scrofa) and Eurasian badger (Meles meles) (Kołodziej-Sobocińska et al. 2014, 2016). Genetic analysis and comparison to Gene Bank sequences indicate systematic status of the species in BPF as $S$. erinaceieuropaei (Liu et al. 1997).

Intermediate hosts for Spirometra sp., such as amphibians and reptiles, are already considered important vectors for parasite transmission in Asia (Wang et al. 2011, 2014; Nelli et al. 2014; Hong et al. 2016). In Europe, the only three cases of infected reptiles, in a common European viper (Vipera berus) and a grass snake (Natrix natrix), have been recorded in Italy, Germany, and Belarus (Joyeux and Baer 1927; Odening et al. 1980; Shimalov et al. 2000).

The aim of our study was the survey of reptiles as hosts of Spirometra sp. and genetic confirmation of the species identification. Current knowledge indicates that reptiles in particular have never been reported as a host of Spirometra sp. in Poland.

\section{Materials and methods}

A total of 59 dead reptiles from Białowieża Primeval Forest (23 ind.) and Biebrza National Park (BNP) (36 ind.) were found and necropsied. These included 53 grass snakes, two sand lizards (Lacerta agilis), two common European vipers, one viviparous lizard (Zootoca vivipara), and one slowworm (Anguis fragilis). Reptiles were sexed, measured, and checked for Spirometra sp. presence. The skin of each individual was slit from the neck to the end of the tail along the spine. Visceral mass was checked for the presence of Spirometra sp. plerocercoids.

To identify a species of isolated larvae we used primers described by Liu et al. (1997). An over $240 \mathrm{bp}$ length sequence of evolutionary conserved nuclear 18S rRNA gene was used for this test. We compared obtained sequences with the ones acquired from three wild boars and three badgers from BPF (Kołodziej-Sobocińska et al. 2014, 2016), as well as from GenBank deposits, using the ClustalW Multiple alignment test. All molecular analyses were performed online using Basic Local Alignment Search Tool (BLAST) (https://blast.ncbi.nlm.nih.gov/Blast.cgi).

\section{Results and discussion}

During the necropsies, we found spargana of Spirometra sp. in two grass snakes from BPF (Fig. 1). Both snakes were adults - one a $32-\mathrm{cm}$-long female found in the forest and the other a 64-cm-long male found in Białowieża village - and were infected by one and three larvae, respectively. In both individuals, spargana were located subcutaneously near the cloaca. The average length of a sparganum was $50 \mathrm{~mm}$ (range $5-120 \mathrm{~mm}$ ).

Due to the lack of specific plerocercoid morphological features, parasite identification was performed using molecular methods. The analyzed $18 \mathrm{~S}$ rRNA gene fragment of larvae isolated from snake tissue showed $99 \%$ identity with the GenBank-deposited Spirometra erinaceieuropaei (KX528100.1) and 100\% identity with Spirometra erinacei (D64072.1) from both GenBank and previously investigated sequences of plerocercoids in badgers and wild boars from BPF (Kołodziej-Sobocińska et al. 2014, 2016) (Fig. 2a, b).

Our finding is the first genetically confirmed record of Spirometra sp. in reptiles in Europe, and the first infected reptiles in Poland. In Asia, where this tapeworm occurs frequently, many species of snakes act as second intermediate hosts (Wang et al. 2011, 2014; Nelli et al. 2014). Reptiles infected with spargana were also recorded in South America (Oda et al. 2016), Australia (Zhu et al. 2002), and Africa (Pantchev and Tappe 2011). Morphological identification of Spirometra sp. plerocercoids is very unreliable. Thus, molecular methods are the most recommended confirmation of their systematic classification (Wongkulab et al. 2011; Kołodziej-Sobocińska et al. 2014, 2016). 

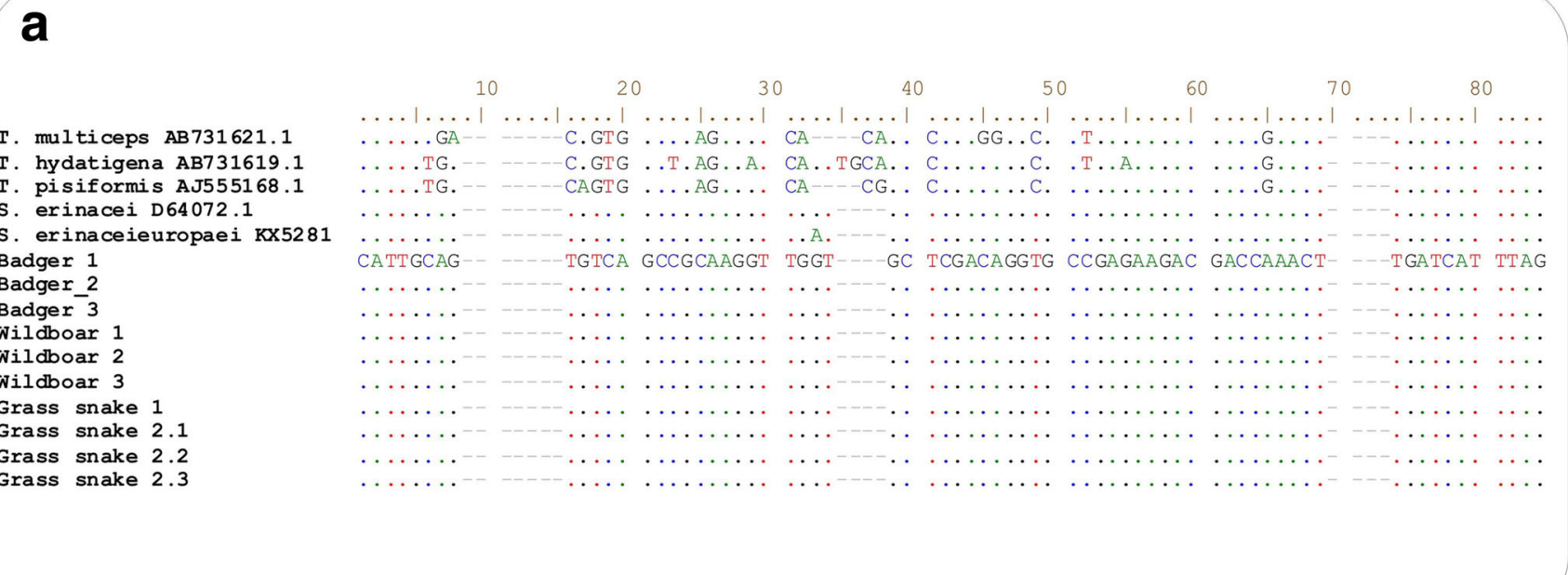

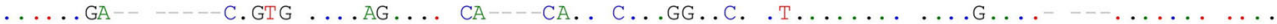

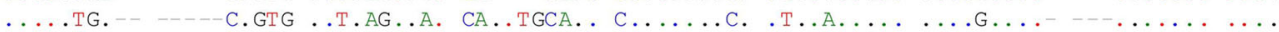

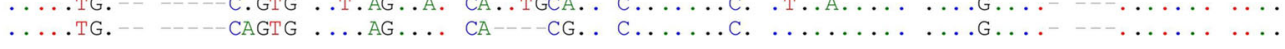

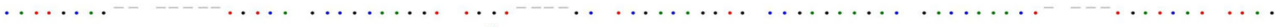

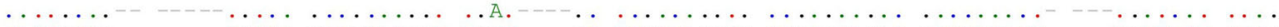

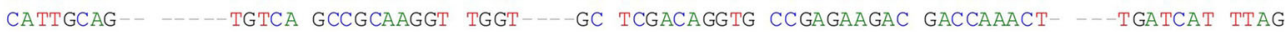

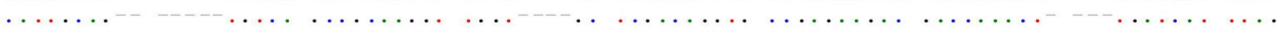

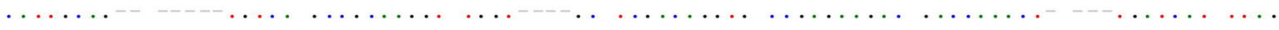

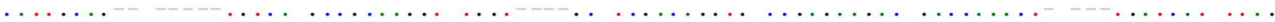

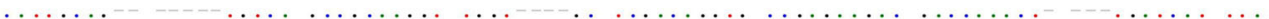

,

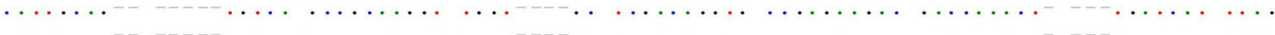

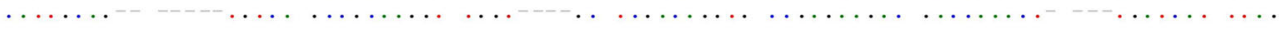

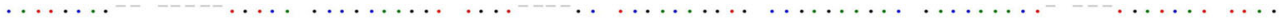

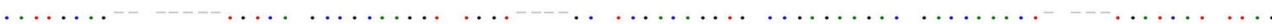

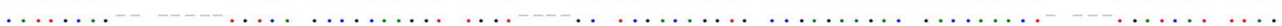

\section{b}

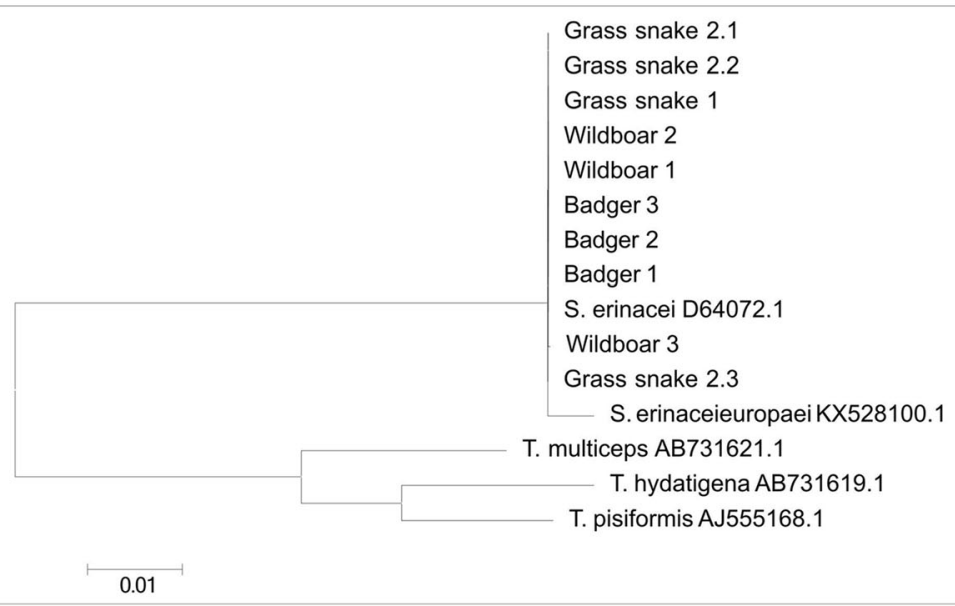

Fig. 2 Results of molecular analysis. a Partial alignment of $240 \mathrm{bp}$ fragments of $18 \mathrm{~S}$ rRNA gene fragments extracted from Spirometra sp. plerocercoids found in grass snakes, badgers, and wild boars, as well as other Cestoda species from NCBI deposits, along with their access IDHere. The reference sequence is Spirometra sp. described by Kołodziej-Sobocińska et al. (2014). Dotted nucleotides are identical to those of a reference. b Molecular phylogenetic analysis of the studied sequences from Fig. 2a, by the maximum likelihood method based on the Tamura-Nei model (Tamura and Nei 1993). The tree is drawn to scale, with branch lengths measured in the number of substitutions per site. Evolutionary analyses were conducted in MEGA6 (Tamura et al. 2013)
Grass snakes are strongly associated with wetlands, where they can most likely become infected with procercoids by swallowing water copepods or consuming amphibians (Luiselli et al. 1997). Moreover, they can be preyed upon by several species of mammals and birds that are known to occasionally feed on reptiles (Jędrzejewska and Jędrzejewski 1998), transferring spargana to subsequent trophic levels. This report also confirms the role of reptiles as parasite transmitters outside Asia and reveals additional routes of sparganosis transmission in European wildlife. Further studies are recommended to provide a deeper explanation of the role of non-mammalian hosts in the spread of Spirometra sp. in the natural environment.

Acknowledgments We would like to thank Mrs. Ewelina Hapunik and Mr. Dariusz Chilecki from the Mammal Research Institute Polish Academy of Sciences, as well as Mr. Woody Janssen from Van Hall Larenstein, University of Applied Sciences, for the help in collecting and dissecting reptiles. Additionally, many volunteers, among others Nikodem Czeremczuk, Eliza Łozowska, and Anna Wójcik, helped in collecting reptiles in the field. We are also grateful to Anita Michalak for English language correction.

Funding information The study was financed by the National Science Centre project no. 2016/21/B/NZ8/02429. 


\section{Compliance with ethical standards}

Conflict of interest The authors declare that there is no conflict of interest.

Open Access This article is distributed under the terms of the Creative Commons Attribution 4.0 International License (http:// creativecommons.org/licenses/by/4.0/), which permits unrestricted use, distribution, and reproduction in any medium, provided you give appropriate credit to the original author(s) and the source, provide a link to the Creative Commons license, and indicate if changes were made.

\section{References}

Bearup AJ (1953) Life history of a Spirometrid tapeworm causing sparganosis in feral pigs. Aust Vet J 29:217-224

Furmaga S (1953) Spirometra janickii sp. n. (Diphyllobothriidae). Acta Parasitol Pol 1:29-59

Hong Q, Feng JP, Liu HJ, Li XM, Gong LR, Yang Z, Yang WM, Liang XF, Zheng RJ, Cui ZC, Wang WL, Chen DX (2016) Prevalence of Spirometra mansoni in dogs, cats, and frogs and its medical relevance in Guangzhou, China. Int J Infect Dis 53:41-45

Jędrzejewska B, Jędrzejewski W (1998) Predation in vertebrate communities. The Białowieża Primeval Forest as a case study. SpringerVerlag, Berlin

Joyeux C, Baer JG (1927) Sur quelques larves de Bothriocephales. Bull Soc Pathol Exot Paris 20:921-937

Kołodziej-Sobocińska M, Miniuk M (2018) Sparganosis - neglected zoonosis and its reservoir in wildlife. Med Weter 74:224-227

Kołodziej-Sobocińska M, Tokarska M, Kowalczyk R (2014) The first report of sparganosis (Spirometra sp.) in Eurasian badger (Meles meles). Parasitol Int 63:397-399

Kołodziej-Sobocińska M, Miniuk M, Ruczyńska I, Tokarska M (2016) Sparganosis in wild boar (Sus scrofa) - implications for veterinarians, hunters, and consumers. Vet Parasitol 227:115-117

Kołodziej-Sobocińska M, Yakovlev Y, Schmidt K, Hurníková Z, Ruczyńska I, Bednarski M, Tokarska M (2018) Update of the helminth fauna in Eurasian lynx (Lynx lynx) in Poland. Parasitol Res 117:2613-2621. https://doi.org/10.1007/s00436-018-5953-0
Liu DW, Kato H, Sugane K (1997) The nucleotide sequence and predicted secondary structure of small subunit (18S) ribosomal RNA from Spirometra erinaceieuropaei. Gene 184:221-227

Luiselli L, Capula M, Shine R (1997) Food habits, growth rates, and reproductive biology of grass snakes, Natrix natrix (Colubridae) in the Italian Alps. J Zool 241:371-380

Nelli S, Felix D, Marine A (2014) Seven new species of helminths for reptiles from Armenia. Acta Parasitol 59:442-447

Oda FH, Borteiro C, da Graça RJ, Tavares LER, Crampet A, Guerra V, Lima FS, Bellay S, Karling LC, Castro O (2016) Parasitism by larval tapeworms genus Spirometra in South American amphibians and reptiles: new records from Brazil and Uruguay, and a review of current knowledge in the region. Acta Trop 164:150-164

Odening K, Tscherner W, Bockhardt I (1980) Experimentelle Bestimmung von Cestodeneiern (Spirometra) aus Goldschakal und Luchs. Milu 5:245-251

Pantchev N, Tappe D (2011) Pentastomiasis and other parasitic zoonoses from reptiles and amphibians. Berl Munch Tierarztl Wochenschr 124:528-535

Shimalov VV, Shimalov VT, Shimalov AV (2000) Helminth fauna of lizards (Reptilia, Sauria) in the southern part of Belarus. Parasitol Res 86:343-343

Tamura K, Nei M (1993) Estimation of the number of nucleotide substitutions in the control region of mitochondrial DNA in humans and chimpanzees. Mol Biol Evol 10:512-526

Tamura K, Stecher G, Peterson D, Filipski A, Kumar S (2013) MEGA6: molecular evolutionary genetics analysis version 6.0. Mol Biol Evol 30:2725-2729

Wang F, Zhou L, Gong S, Deng Y, Zou J, Wu J, Liu W, Hou F (2011) Severe infection of wild-caught snakes with Spirometra erinaceieuropaei from food markets in Guangzhou, China involves a risk for zoonotic sparganosis. J Parasitol 97:170-171

Wang F, Li W, Hua L, Gong S, Xiao J, Hou F, Ge Y, Yang G (2014) Spirometra (Pseudophyllidea, Diphyllobothriidae) severely infecting wild-caught snakes from food markets in Guangzhou and Shenzhen, Guangdong, China: implications for public health. Sci World J 2014:1-5. https://doi.org/10.1155/2014/874014

Wongkulab P, Sukontason K, Chaiwarith R (2011) Sparganosis: a brief review. J Infect Dis Antimicrob Agents 28:77-80

Zhu X, Beveridge I, Berger L, Barton D, Gasser R (2002) Singlestrand conformation polymorphism-based analysis reveals genetic variation within Spirometra erinacei (Cestoda: Pseudophyllidea) from Australia. Mol Cell Probes 16:159-165 